GSA DATA REPOSITORY 2017099

Liu and $\mathrm{Wu}$ et al.

Zinc isotope evidence for intensive magmatism immediately before the end-Permian mass extinction

Sheng-Ao Liu", Huaichun Wu ${ }^{*}$, Shu-zhong Shen, Ganqing Jiang, Shihong Zhang, Yiwen Lv, Hua Zhang, and Shuguang Li

*E-mails: 1sa@cugb.edu.cn; whcgeo@cugb.edu.cn

This file includes:

1. SAMPLING SECTION

2. ANALYTICAL METHODS

3. Figures DR1-4

4. Tables DR1-5

5. References 


\section{SAMPLING SECTION}

The Meishan section $\left(31^{\circ} 4^{\prime} 55^{\prime \prime} \mathrm{N}, 119^{\circ} 42^{\prime} 22.9^{\prime \prime} \mathrm{E}\right)$ is the most intensively studied Permian/Triassic boundary (PTB) section and is located in Changxing County, Zhejiang Province, South China. Paleogeographically, it was located near the tropics during the Late Permian and Early Triassic (Yin et al., 2001; Jin et al., 2006) (Fig. 1). The Meishan section is the stratotype section for the Changhsingian Stage, the Global Stratotype Section and Point (GSSP) for the PTB, and the Wuchiapingian-Changhsingian boundary (WCB) (Yin et al., 2001; Jin et al., 2006).

Core M1 was drilled $500 \mathrm{~m}$ west of the Meishan section D. Recovered Upper Permian-Lower Triassic strata correlate bed-by-bed with the lithostratigraphic units at the outcrops. The rocks of this drill core were considered to be free from outcrop contamination, and may afford the best possible geochemical record for the environmental evolution (Cao et al., 2009). Sixty-five bulk samples of micritic limestone and argillaceous limestone were collected over a 20-m-thick interval (Beds 19-37) across the PTB from Core M1 for carbon and zinc isotope analysis (Fig. 2, Tables DR1 and DR2). Beds 19-24 of the Changhsing Formation (Upper Permian) are composed of bioclastic and micritic limestone with thinly interbedded chert layers. Beds 25-37 of the Yinkeng Formation (uppermost Permian to lowermost Triassic) consist of parallel-laminated calcareous mudstone and thin-bedded argillaceous limestone. These rocks were deposited in middle-upper slope environments (Yin et al., 2001; Jin et al., 2006).

Conodont biostratigraphy has been intensively studied at the Meishan section (Yin et al., 2001; Jin et al., 2006; Jiang et al., 2007; Zhang et al., 2009; Yuan et al., 2014). The conodont zones in the studied interval (Beds 19-37), in ascending order, include $C$. changxingensis Zone (Beds 19-22), C. yini Zone (Beds 22-24d), C. meishanensis Zone (Beds 24e-25), H. praeparvus-H. changxingensis Zone (Beds 26-27b), H. parvus Zone 
(Bed 27c), I. staeschei Zone (Beds 27d-28), I. isarcica Zone (Bed 29), and C. planata Zone (Beds 30-37). The GSSP for the PTB was defined at the first appearance datum (FAD) of $H$. parvus (Yin et al., 2001). The mass extinction interval was identified between the top of Bed 24e and Bed 28.

Recently, Burgess et al. (2014) re-dated the zircons from five volcanic ash beds of the Meishan section using high-precision U-Pb ID-TIMS method, and the new ages are $251.495 \pm 0.064 \mathrm{Ma}$ (Bed 34), 251.583 $\pm 0.086 \mathrm{Ma}($ Bed 33), $251.880 \pm 0.031 \mathrm{Ma}($ Bed 28), $251.941 \pm 0.037 \mathrm{Ma}$ (Bed 25) and $252.104 \pm 0.089 \mathrm{Ma}$ (Bed 22) (Fig. 2). These ages are consistent with previously published ages (Shen et al., 2011) and the astrochronology (Wu et al., 2013) within error. The ages and durations of the Zn isotope stages (S1-S4 in Fig. 2) were determined by the U-Pb ages (Burgess et al., 2014) and astronomical time scale (Wu et al., 2013).

\section{ANALYTICAL METHODS}

\subsection{Carbon and oxygen isotope analyses of carbonates}

Bulk carbonate samples were powdered to react with purified $\mathrm{H}_{3} \mathrm{PO}_{4}$ under vacuum at $50{ }^{\circ} \mathrm{C}$ for $12 \mathrm{~h}$. Generated $\mathrm{CO}_{2}$ gas was cryogenically extracted and sealed in vacuumed tubes for isotope analysis. Isotope analyses were carried out at the Nanjing Institute of Geology and Palaeontology, Chinese Academy of Sciences using a Finnigan MAT-253 isotope-ratio mass spectrometer. Reproducibility on replicated samples is better than $0.03 \%$ (2s) for $\delta^{13} \mathrm{C}_{\text {carb }}$, and $0.08 \%$ (2s) for $\delta^{18} \mathrm{O}$. Peedee Belemnite (PDB) calibration was made through an internal laboratory standard GBW04405 with $\delta^{13} \mathrm{C}_{\text {carb }}=$ $0.57 \%$ and $\delta^{18} \mathrm{O}=-8.49 \%$. The $\delta^{13} \mathrm{C}_{\text {carb }}$ profile obtained in this study is consistent with that of Shen et al. (2011) and Cao et al. (2009) (Fig. 2; Tables DR1-2).

\subsection{Carbonate dissolution procedure for $\mathrm{Zn}$ isotopes and concentrations}

A leaching procedure modified from previously established methods (Pichat et al., 
2003; Kunzmann et al., 2013) was designed to extract the carbonate fraction of samples. This chemical procedure allows the separation of the carbonate fraction from other components without inducing $\mathrm{Zn}$ isotope fractionation (Pichat et al., 2003). Samples were carefully checked to avoid visible veins and fractures. Sample powders of 200 mesh were obtained using an agate mortar that is carefully cleaned with ethanol and Milli-Q water (18.2 M $\Omega$ ). The resulting powders were then thoroughly homogenized. The whole procedure avoids $\mathrm{Zn}$ contamination by metal tools. The leaching steps were performed in a clean room under laminar flow hood (class 100) to minimize $\mathrm{Zn}$ contamination. All reagents have been detected to ensure no potential $\mathrm{Zn}$ contamination. The supplies used in the leaching experiments were cleaned thoroughly between each sample. Teflon perfluoroalkoxy (PFA) beakers were used for leaching solution, which were pre-cleaned with 1:1 (v/v) nitric acid and hydrochloric acid. Polypropylene filters and centrifuge tubes were soaked in $10 \%$ nitric acid over 24 hours without heating. Hydrosoluble salts and exchangeable fraction on clays were removed before leaching of carbonates using Milli-Q water and $1 \mathrm{~N}$ ammonium acetate $\left(\mathrm{NH}_{4} \mathrm{AC}\right)$ alternately.

The carbonate fraction was then leached with two steps of $0.05 \mathrm{M}$ super-pure acetic acid in a thermostat at $65{ }^{\circ} \mathrm{C}$, until no bubbles were generated. The supernatants were collected after $20 \mathrm{~min}$ of centrifugation, followed by filtration. The leached supernatants were dried at $80^{\circ} \mathrm{C}$ and added with $2 \mathrm{ml}$ of $8 \mathrm{M} \mathrm{HCl}$. This step was repeated twice to remove acetic acid. Finally, the solution was prepared in $1 \mathrm{ml}$ of $8 \mathrm{M} \mathrm{HCl}$ for chemical anion-exchange separation. During the preparation, blanks (including reagents and containers) are added to mimic the leaching procedure. The leaching procedure ensures the separation of the carbonate fraction of samples without contamination from other components (such as clays, salts, and oxides). This is consistent with the extremely high $(>10,000)$ molar $\mathrm{Ca} / \mathrm{Al}$ ratio of leached solution and $<0.1 \%$ difference in $\delta^{66} \mathrm{Zn}$ of duplicate analysis. Most samples were also leached following previously established 
method (Pichat et al., 2003; Kunzmann et al., 2013) using 1.5 M acetic acid and no significant differences were found. However, the weaker acetic acid $(0.05 \mathrm{M})$ used in this study faithfully reduce the risk of release of $\mathrm{Zn}$ from other components such as clays and oxides. In particular, the two steps of leaching on the same samples could help to assess whether a single component (carbonate) was leached and no other components were significantly involved. This is achieved by analyzing $\mathrm{Zn}$ isotope compositions of the two leachates from the same samples.

\subsection{Zinc isotope measurements}

The column chemistry and instrumental analysis of $\mathrm{Zn}$ isotopes follow the methods of Liu et al. (2014) and Maréchal et al. (1999). The leaching solutions were dried at $80{ }^{\circ} \mathrm{C}$. For some samples, the leaching residues were completely dissolved in a 1:1 (v/v) mixture of double-distilled $\mathrm{HF}$ and $\mathrm{HNO}_{3}$ in Savillex screw-top beakers. Zinc was purified by an ion-exchange chromatography using Bio-Rad strong anion resin AG-MP-1M. $2 \mathrm{ml}$ pre-cleaned resin was loaded onto the column. Matrix elements were eluted in the first 10 $\mathrm{ml} 8 \mathrm{~N} \mathrm{HCl}$. Copper and iron were then collected in the following $24 \mathrm{ml}$ of $8 \mathrm{~N} \mathrm{HCl}+$ $0.001 \% \mathrm{H}_{2} \mathrm{O}_{2}$ and $18 \mathrm{ml}$ of $2 \mathrm{~N} \mathrm{HCl}$. Zinc was collected in the subsequent $15 \mathrm{ml}$ of $0.5 \mathrm{~N}$ $\mathrm{HNO}_{3}$. This procedure allows $\mathrm{Ca}$ to be completely separated from $\mathrm{Zn}$ and a $100 \%$ recovery for $\mathrm{Zn}$. The total $\mathrm{Zn}$ of procedural blanks is $2 \mathrm{ng}$, which is less than $0.5 \%$ of loaded $\mathrm{Zn}(>0.4 \mu \mathrm{g})$ in the studied samples. The $\mathrm{Zn}$ fractions were repeatedly dried and dissolved with $3 \% \mathrm{HNO}_{3}$ to remove all chlorine prior to isotope analysis.

Zinc isotopic ratios were measured using a Neptune plus multi-collector inductively coupled plasma mass-spectrometry (MC-ICP-MS) at the Isotope Geochemistry Laboratory of the China University of Geosciences (Beijing). Sample-standard bracketing method was used to correct for instrumental mass fractionation. The samples and standards are run in $\sim 200 \mathrm{ppb}$ diluted solution dissolved in $3 \%$ (v) $\mathrm{HNO}_{3}$. The take-up 
time is $80 \mathrm{~s}$. The high-sensitivity $(\mathrm{X})$ cones made of Ni are used for the increase of transmission to ensure that the ${ }^{64} \mathrm{Zn}$ signals are usually at the level of $\sim 1.5 \mathrm{~V} / 100 \mathrm{ppb}$. Zinc isotopic data are reported in standard $\delta$-notation in per mil relative to standard reference material JMC 3-0749L by Maréchal et al. (1999), the Johnson Matthey (JMC) $\mathrm{Zn}$ standard solution from the Lyon-CNRS laboratory. The long-term external reproducibility for $\delta^{66} \mathrm{Zn}$ measurements of synthetic pure $\mathrm{Zn}$ solutions is better than $\pm 0.03 \%$ o (2SD) (Fig. DR3), and for natural rock samples the reproducibility is better than $\pm 0.06 \%$ o (2SD) based on whole procedure repeat of the standard materials ( $\mathrm{Lv}$ et al., 2016). The error of reported $\delta^{66} \mathrm{Zn}$ values represents replicate measurements of the same samples, which is always used while greater than the long-term reproducibility of $\pm 0.06 \%$. The $\mathrm{Zn}$ isotope data for leaching solutions and residues are reported in Table DR2. Zn isotope data of the leachatesare the mean of the two steps of leaching on the sample samples, which agree with each other within $\pm 0.1 \%$. International rock standards (BHVO-2, BIR-1a) were analyzed during the course of this study, yielding $\delta^{66} \mathrm{Zn}$ values of $0.32 \pm$ $0.04 \%$ and $0.28 \pm 0.04 \%$ respectively (Table DR3), which agree with those reported by previous studies $(0.28 \pm 0.12 \%$ and $0.26 \pm 0.11 \%$, respectively; e.g., Moeller et al., 2012). All samples analyzed in this study yield a slope of 1.996 in a $\delta^{68} \mathrm{Zn}-\delta^{66} \mathrm{Zn}$ cross-plot, which is consistent with the mass-dependent line with a slope of 2 , indicating mass-dependent $\mathrm{Zn}$ isotope fractionation and no analytical artifacts from unresolved isobaric interferences on $\mathrm{Zn}$ isotopes.

\subsection{Major and trace element analysis of bulk rocks and leaching solutions}

The carbonate fractions were extracted for $\mathrm{Zn}$ concentration analysis following the same procedure for $\mathrm{Zn}$ isotope measurement (Table DR4). Trace element analyses of the leached solutions were accomplished using an inductively coupled plasma mass spectrometer (ICP-MS) similar to previously reported procedures (Liu et al., 2010). Reproducibility was better than $5 \%$ for elements with concentrations $>10 \mathrm{ppm}$ and less 
than $10 \%$ for those $<10 \mathrm{ppm}$ based on long-term analysis of standard materials. The basalt standard (BHVO-2) and carbonatite standard (COQ-1) analyzed yield Zn concentrations of $107 \mu \mathrm{g} / \mathrm{g}$ and $85 \mu \mathrm{g} / \mathrm{g}$, respectively, consistent within errors with the recommended values $(103 \mu \mathrm{g} / \mathrm{g}$ and $87 \mu \mathrm{g} / \mathrm{g})$. The $\mathrm{Zn}$ concentration in carbonates is calculated from $\mathrm{Zn}$ contents $(\mu \mathrm{g})$ in the leaching solutions divided by the contents of carbonates $(\mathrm{g})$, the latter of which are calculated based on major elements in the bulk rocks.

Some samples of bulk sediments were selected for major element analysis to evaluate the possible influence of silicate components on $\mathrm{Zn}$ isotopic compositions of the leached carbonate fractions (Tables DR5). Major elements were analyzed using the wet-chemistry methods at the China University of Geosciences (Beijing). Losses of ignition (LOI) were determined by gravimetric methods. Analytical reproducibility of major elements is better than $2 \%$ and for the majority is better than $1 \%$.

\subsection{Mass balance calculation on $\mathrm{Zn}$ cycling in the end-Permian ocean}

The global oceanic mass balance of $\mathrm{Zn}$ isotopes was recently evaluated (Little et al., 2014; Vance et al., 2016). The main inputs of $\mathrm{Zn}$ to the modern ocean include rivers, hydrothermal fluids and aeolian dust and the outputs include Fe-Mn oxides and carbonates as well as euxinic sediments. Assuming steady state (within the ca. 50 k.y. residence time of $\mathrm{Zn}$ ) (Little et al., 2014) and the possible external sources (e.g., increased weathering, volcanism or hydrothermal input), the isotope mass balance gives the following equation:

$$
\delta^{66} \mathrm{Zn}_{\text {target }} \times \mathrm{Zn}_{\text {target }}=\delta^{66} \mathrm{Zn}_{\text {initial }} \times \mathrm{Zn}_{\text {initial }}+\delta^{66} \mathrm{Zn}_{\text {addition }} \times \mathrm{Zn}_{\text {addition }}(1)
$$

Where:

$\delta^{66} \mathrm{Zn}_{\text {target }}$ and $\mathrm{Zn}_{\text {target }}$ are the $\mathrm{Zn}$ isotope value and oceanic $\mathrm{Zn}$ inventory at the negative $\delta^{66} \mathrm{Zn}$ excursion (Stage S2; Fig. 2). We use the measured $\delta^{66} \mathrm{Zn}$ of the Meishan 
section to approximate the oceanic $\delta^{66} \mathrm{Zn}\left(\delta^{66} \mathrm{Zn}_{\text {target }} \approx 0.34 \%\right)$, and $\mathrm{Zn}_{\text {target }}=\mathrm{Zn}_{\text {initial }}+$ $\mathrm{Zn}_{\text {addition }}$.

$\delta^{66} \mathrm{Zn}_{\text {initial }}$ and $\mathrm{Zn}_{\text {initial }}$ are the oceanic $\delta^{66} \mathrm{Zn}$ and oceanic $\mathrm{Zn}$ inventory before the negative $\delta^{66} \mathrm{Zn}$ shift (Stage S1 in Fig. 2). Because the $\delta^{66} \mathrm{Zn}$ values $(\sim 0.83 \%$ ) before the negative shift in Meishan section is similar to that of the modern carbonates $\left(\delta^{66} \mathrm{Zn} \approx\right.$ 0.9\%; Little et al., 2014), we assume that the average global ocean $\mathrm{Zn}$ inventory and isotope values before the negative $\delta^{66} \mathrm{Zn}$ shift were also similar to those of the modern ocean $\left(\mathrm{Zn}_{\text {initial }} \approx 7.3 \times 10^{12} \mathrm{~mol}, \delta^{66} \mathrm{Zn}_{\text {initial }} \approx 0.51 \%\right.$; Little et al., 2014).

$\delta^{66} \mathrm{Zn}_{\text {addition }}$ and $\mathrm{Zn}_{\text {addition }}$ are the $\mathrm{Zn}$ isotope value and mass of external input (i.e., weathering or riverine input, direct volcanic or hydrothermal input into the ocean).

Assuming that the negative $\delta^{66} \mathrm{Zn}$ shift was caused by $\mathrm{CO}_{2}$-driven weathering input that has a $\delta^{66} \mathrm{Zn}_{\text {addition }} \approx 0.33 \%$ (Little et al., 2014), our calculation indicates that:

$$
\begin{aligned}
\mathrm{Zn}_{\text {addition }} & =\left[\left(\delta^{66} \mathrm{Zn}_{\text {target }} \times \mathrm{Zn}_{\text {initial }}\right)-\left(\delta^{66} \mathrm{Zn}_{\text {initial }} \times \mathrm{Zn}_{\text {initial }}\right)\right] /\left(\delta^{66} \mathrm{Zn}_{\text {addition }}-\delta^{66} \mathrm{Zn}_{\text {target }}\right) \\
& =\left[0.34 \times\left(7.3 \times 10^{12}\right)-0.51 \times\left(7.3 \times 10^{12}\right)\right] /(0.33-0.34) \\
& =1.24 \times 10^{14} \mathrm{~mol}
\end{aligned}
$$

With the modern riverine input of $5.9 \times 10^{8} \mathrm{~mol} / \mathrm{yr}$, even if the weathering input was 10 times of the modern riverine input, the amount of time needed to lower the $\delta^{66} \mathrm{Zn}$ down to crustal value, as recorded in the Meishan section, would take 20,000 years. The abrupt $\delta^{66} \mathrm{Zn}$ shift in Meishan happened at a $\leq 3 \mathrm{~cm}$ interval that has been constrained as $<1,500$ years (Wu et al., 2013; Burgess et al., 2014). In such a time framework, weathering input alone may not be able to shift $\delta^{66} \mathrm{Zn}$ down to the crustal value. A more ${ }^{66} \mathrm{Zn}$-depleted source is needed. Isotopically light $\mathrm{Zn}$ sources from volcanic ashes, hydrothermal fluids and/or extremely fast weathering of fresh large igneous provinces (LIPs) are needed. 


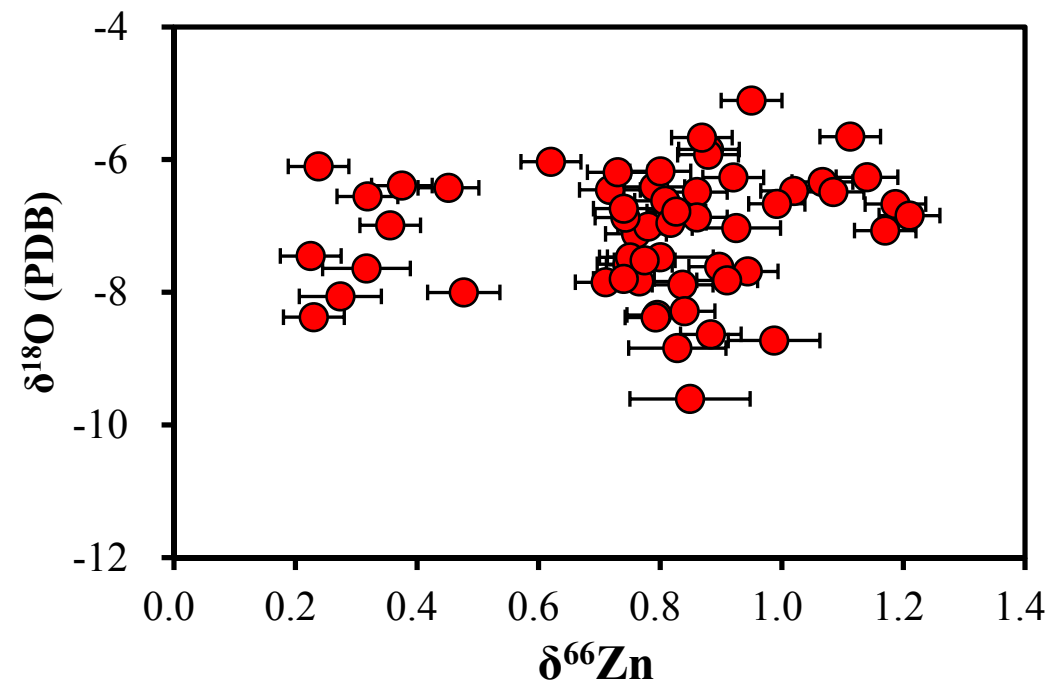

Figure DR1. Cross plot of $\delta^{66} \mathrm{Zn}-\delta^{18} \mathrm{O}$ of carbonates showing the absence of covariation. The lack of $\delta^{66} \mathrm{Zn}-\delta^{18} \mathrm{Oand} \delta^{66} \mathrm{Zn}-\mathrm{Sr} / \mathrm{Ca}$ (Fig. 3 in main text) covariance indicates that the $\mathrm{Zn}$ isotope shift is not related to precipitation rate or diagenesis. 


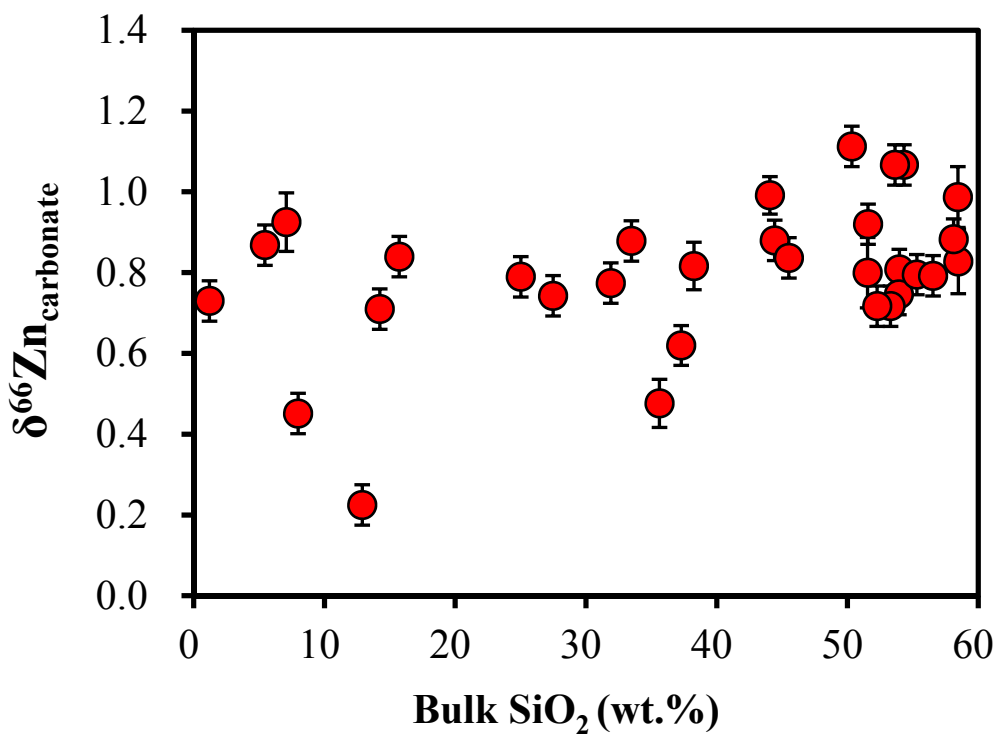

Figure DR2. Cross plot of $\delta^{66} \mathrm{Zn}$ vs. $\mathrm{SiO}_{2}$ of bulk-sediments from the Meishan section. The absence of correlation suggests that the temporal $\delta^{66} \mathrm{Zn}$ variations are not caused by adsorption of $\mathrm{Zn}$ onto silicate or clay phases. 

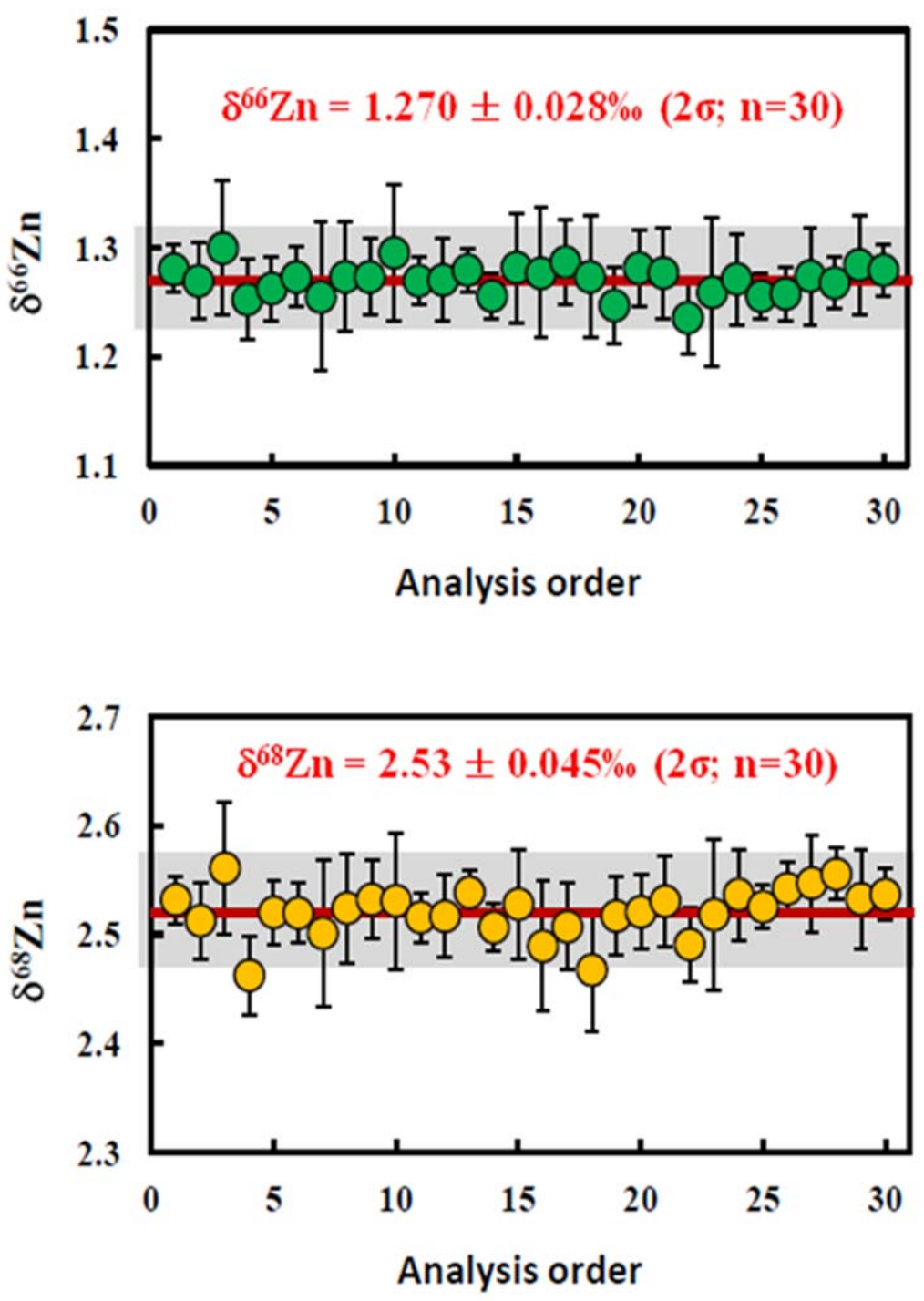

Figure DR3. Long-term analysis of in-house zinc standard solutions with reproducibility of $\pm 0.028 \%$ ( $2 \mathrm{SD}$ ) for $\delta^{66} \mathrm{Zn}$ measurements. 


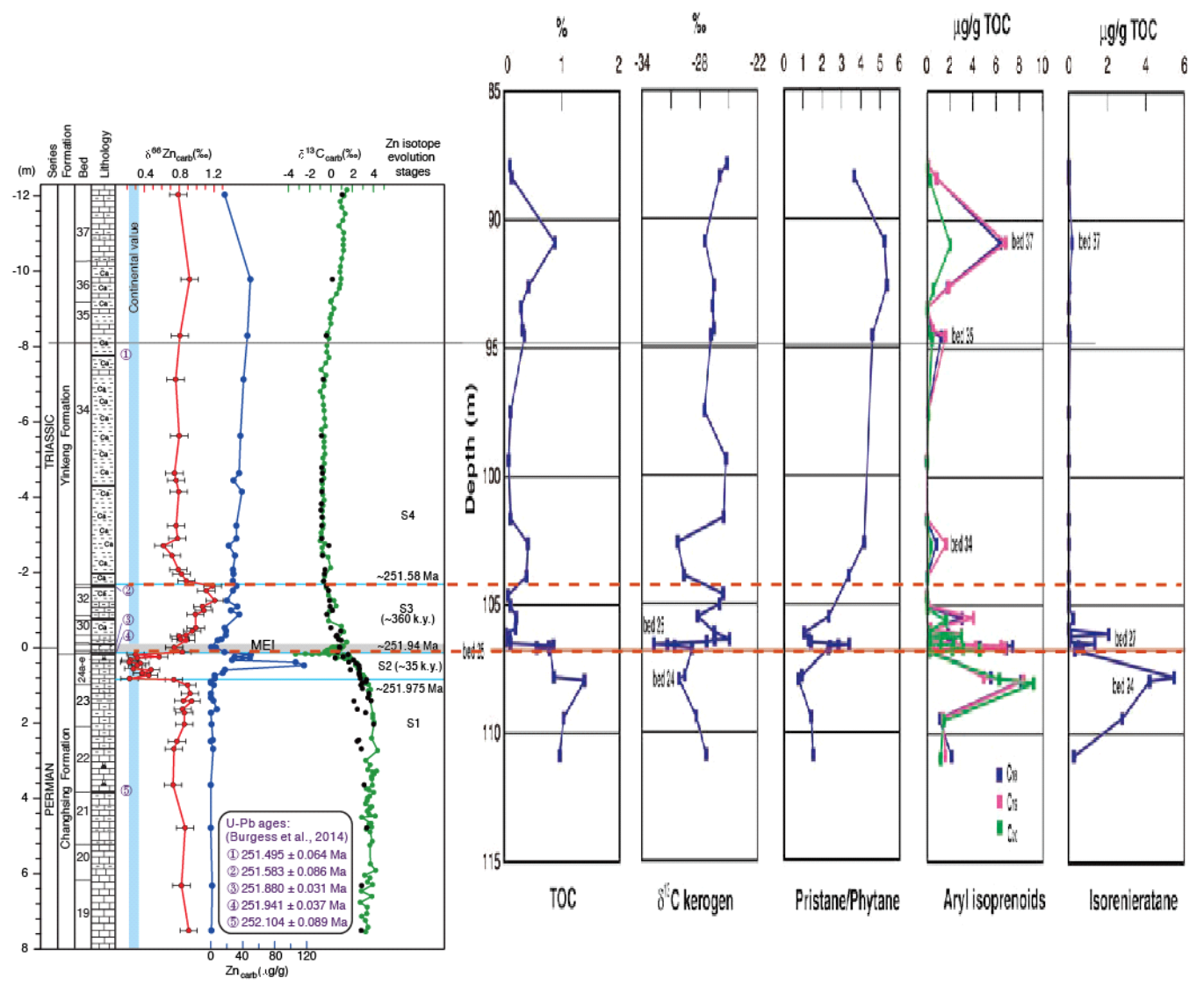

Figure DR4. Comparison between $\mathrm{Zn}$ isotope data in this study and organic geochemical, biomarker, and bulk carbon isotopic data from Grice et al. (2005). 
Table DR1. Carbonate carbon and oxygen isotope data across the PTB from Meishan, South China.

\begin{tabular}{|c|c|c|c|c|c|}
\hline $\begin{array}{l}\text { Depth to } \\
\text { PTB (m) }\end{array}$ & Lithology & $\begin{array}{c}\delta^{13} \mathrm{C}_{\mathrm{PDB}} \\
(\% \mathrm{o})\end{array}$ & $\begin{array}{l}\delta^{18} \mathrm{O} \\
(\% 0)\end{array}$ & $\begin{array}{l}\text { Depth to PTB } \\
\text { (m) }\end{array}$ & $\begin{array}{c}\delta^{13} \mathrm{C}_{\mathrm{PDB}} \\
(\% \mathrm{\%})\end{array}$ \\
\hline This Study & & & \multicolumn{3}{|c|}{ Shen et al. (2011) } \\
\hline-12.03 & Argillaceous limestone & 1.06 & -6.41 & -12.16 & 1.51 \\
\hline-9.78 & Micritic limestone & 0.14 & -6.27 & -12.00 & 1.23 \\
\hline-8.29 & Argillaceous limestone & -0.43 & -6.62 & -11.85 & 0.88 \\
\hline-7.13 & Argillaceous limestone & -0.72 & -7.12 & -11.69 & 1.03 \\
\hline-5.63 & Argillaceous limestone & -0.88 & -7.47 & -11.53 & 1.34 \\
\hline-4.79 & Argillaceous limestone & -0.87 & -7.78 & -11.35 & 1.12 \\
\hline-4.63 & Argillaceous limestone & -0.80 & -7.58 & -11.20 & 0.76 \\
\hline-4.45 & Argillaceous limestone & -0.87 & -7.59 & -11.02 & 1.21 \\
\hline-4.14 & Argillaceous limestone & -0.85 & -8.34 & -10.84 & 1.18 \\
\hline-3.82 & Argillaceous limestone & -0.89 & -6.27 & -10.70 & 1.16 \\
\hline-3.66 & Argillaceous limestone & -0.94 & -7.07 & -10.55 & 1.19 \\
\hline-3.47 & Argillaceous limestone & -0.83 & -6.47 & -10.34 & 1.03 \\
\hline-3.24 & Argillaceous limestone & -0.86 & -7.84 & -10.12 & 0.91 \\
\hline-2.90 & Argillaceous limestone & -0.86 & -7.01 & -9.98 & 0.85 \\
\hline-2.71 & Argillaceous limestone & -0.19 & -6.03 & -9.80 & 0.96 \\
\hline-2.45 & Argillaceous limestone & -0.79 & -6.46 & -9.65 & 0.88 \\
\hline-2.07 & Argillaceous limestone & -0.56 & -8.38 & -9.54 & 0.79 \\
\hline-1.96 & Argillaceous limestone & -0.60 & -8.84 & -9.40 & 0.54 \\
\hline-1.77 & Argillaceous limestone & -0.68 & -8.63 & -9.22 & 0.04 \\
\hline-1.64 & Argillaceous limestone & -0.42 & -6.67 & -9.18 & -0.02 \\
\hline-1.52 & Argillaceous limestone & -0.23 & -5.65 & -9.00 & 0.28 \\
\hline-1.25 & Argillaceous limestone & -0.13 & -6.84 & -8.86 & 0.06 \\
\hline-1.10 & Argillaceous limestone & -0.08 & -6.34 & -8.75 & -0.08 \\
\hline-0.99 & Argillaceous limestone & 0.15 & -6.49 & -8.61 & 0.00 \\
\hline-0.89 & Argillaceous limestone & -0.39 & -8.73 & -8.36 & -0.32 \\
\hline-0.53 & Argillaceous limestone & 0.00 & -6.67 & -8.21 & -0.18 \\
\hline-0.45 & Argillaceous limestone & 0.92 & -5.11 & -8.03 & -0.37 \\
\hline-0.33 & Argillaceous limestone & 0.57 & -5.85 & -7.85 & -0.38 \\
\hline-0.31 & Argillaceous limestone & 0.49 & -6.18 & -7.71 & -0.19 \\
\hline-0.23 & Argillaceous limestone & 0.72 & -6.95 & -7.56 & -0.47 \\
\hline-0.20 & Argillaceous limestone & 0.96 & -5.92 & -7.38 & -0.93 \\
\hline-0.02 & Dolomitic limestone & 0.81 & -6.17 & -7.24 & -0.46 \\
\hline-0.01 & Dolomitic limestone & 0.84 & -6.87 & -7.09 & -0.63 \\
\hline
\end{tabular}




\begin{tabular}{llllll}
\hline 0.12 & Argillaceous limestone & 0.64 & -7.89 & -6.95 & -0.66 \\
0.18 & Micritic limestone & 1.62 & -7.47 & -6.81 & -1.00 \\
0.24 & Micritic limestone & 1.16 & -7.85 & -6.66 & -0.77 \\
0.27 & Micritic limestone & 1.82 & -6.55 & -6.44 & -0.70 \\
0.33 & Micritic limestone & 2.13 & -6.10 & -6.30 & -0.64 \\
0.38 & Micritic limestone & 1.68 & -7.45 & -6.08 & -0.60 \\
0.41 & Micritic limestone & 2.37 & -6.99 & -5.88 & -0.53 \\
0.47 & Micritic limestone & 2.63 & -7.64 & -5.80 & -0.88 \\
0.56 & Micritic limestone & 2.68 & -8.06 & -5.62 & -0.64 \\
0.59 & Micritic limestone & 1.84 & -8.00 & -5.47 & -0.65 \\
0.67 & Micritic limestone & 2.64 & -6.39 & -5.33 & -0.65 \\
0.73 & Micritic limestone & 2.70 & -6.42 & -5.15 & -0.56 \\
0.82 & Micritic limestone & 2.78 & -8.37 & -5.04 & -0.74 \\
0.85 & Micritic limestone & 3.35 & -6.74 & -4.89 & -0.62 \\
0.90 & Micritic limestone & 3.29 & -7.71 & -4.71 & -0.69 \\
0.93 & Micritic limestone & 2.94 & -6.98 & -4.68 & -0.71 \\
0.99 & Micritic limestone & 2.83 & -7.61 & -4.50 & -0.66 \\
1.08 & Micritic limestone & 2.97 & -9.16 & -4.35 & -0.81 \\
1.22 & Micritic limestone & 3.58 & -7.03 & -4.21 & -0.79 \\
1.33 & Micritic limestone & 3.49 & -8.28 & -4.07 & -0.76 \\
1.41 & Micritic limestone & 3.79 & -9.61 & -3.92 & -0.64 \\
1.42 & Micritic limestone & 2.17 & -7.68 & -3.78 & -0.75 \\
1.63 & Micritic limestone & 2.48 & -8.28 & -3.63 & -0.81 \\
1.73 & Micritic limestone & 3.27 & -6.49 & -3.45 & -0.77 \\
2.03 & Micritic limestone & 4.03 & -6.87 & -3.27 & -0.72 \\
2.45 & Micritic limestone & 2.63 & -7.29 & -3.18 & -0.76 \\
2.48 & Micritic limestone & 2.48 & -7.52 & -3.06 & -0.91 \\
2.69 & Micritic limestone & 2.87 & -7.80 & -2.95 & -0.88 \\
3.64 & Micritic limestone & 3.12 & -6.19 & -2.84 & -1.00 \\
4.78 & Micritic limestone & 3.37 & -5.67 & -2.77 & -0.56 \\
6.32 & Micritic limestone & 2.90 & -6.78 & -2.66 & -0.83 \\
7.51 & Micritic limestone & 2.86 & -7.82 & -2.52 & -0.78 \\
\hline & & & & &
\end{tabular}


Table DR2. Zinc isotopic ratios of leached carbonate fraction of samples from the Meishan section, South China

\begin{tabular}{|c|c|c|c|c|c|c|c|}
\hline Bed no. & $\begin{array}{l}\text { Depth to } \\
\text { PTB (m) }\end{array}$ & Lithology & $\begin{array}{c}\delta^{66} \mathrm{Zn} \\
(\% \mathrm{o})\end{array}$ & $2 \mathrm{sd}$ & $\begin{array}{l}\delta^{68} \mathrm{Zn} \\
(\% \text { ) }\end{array}$ & $2 \mathrm{sd}$ & $\delta^{68} \mathrm{Zn} / \delta^{66} \mathrm{Zn}$ \\
\hline 37 & -12.03 & Argillaceous limestone & 0.79 & 0.05 & 1.60 & 0.07 & 2.03 \\
\hline 36 & -9.78 & Micritic limestone & 0.92 & 0.05 & 1.85 & 0.06 & 2.01 \\
\hline 35 & -8.29 & Argillaceous limestone & 0.81 & 0.05 & 1.61 & 0.05 & 1.99 \\
\hline 34 & -7.13 & Argillaceous limestone & 0.76 & 0.05 & 1.53 & 0.09 & 2.01 \\
\hline 34 & -5.63 & Argillaceous limestone & 0.80 & 0.09 & 1.59 & 0.10 & 1.99 \\
\hline 34 & -4.63 & Argillaceous limestone & 0.75 & 0.05 & 1.43 & 0.07 & 1.92 \\
\hline 34 & -4.45 & Argillaceous limestone & 0.77 & 0.05 & 1.48 & 0.07 & 1.94 \\
\hline Residue & -4.45 & Argillaceous limestone & 0.36 & 0.04 & 0.73 & 0.06 & 2.03 \\
\hline 34 & -4.14 & Argillaceous limestone & 0.80 & 0.05 & 1.56 & 0.03 & 1.97 \\
\hline 34 & -3.24 & Argillaceous limestone & 0.77 & 0.06 & 1.52 & 0.10 & 1.99 \\
\hline 34 & -2.90 & Argillaceous limestone & 0.78 & 0.05 & 1.56 & 0.06 & 2.00 \\
\hline 34 & -2.71 & Argillaceous limestone & 0.62 & 0.05 & 1.27 & 0.01 & 2.04 \\
\hline 34 & -2.45 & Argillaceous limestone & 0.72 & 0.05 & 1.51 & 0.02 & 2.11 \\
\hline 34 & -2.07 & Argillaceous limestone & 0.79 & 0.05 & 1.55 & 0.12 & 1.96 \\
\hline 34 & -1.96 & Argillaceous limestone & 0.83 & 0.08 & 1.62 & 0.13 & 1.95 \\
\hline 34 & -1.77 & Argillaceous limestone & 0.88 & 0.05 & 1.77 & 0.05 & 2.01 \\
\hline 32 & -1.64 & Argillaceous limestone & 1.19 & 0.05 & 2.29 & 0.02 & 1.93 \\
\hline 32 & -1.52 & Argillaceous limestone & 1.11 & 0.05 & 2.16 & 0.05 & 1.94 \\
\hline Residue & -1.52 & Argillaceous limestone & 0.32 & 0.04 & 0.63 & 0.06 & 1.97 \\
\hline 32 & -1.25 & Argillaceous limestone & 1.21 & 0.05 & 2.63 & 0.00 & 2.17 \\
\hline 32 & -1.10 & Argillaceous limestone & 1.07 & 0.05 & 2.07 & 0.10 & 1.94 \\
\hline 32 & -0.99 & Argillaceous limestone & 1.08 & 0.05 & 2.10 & 0.03 & 1.93 \\
\hline 32 & -0.89 & Argillaceous limestone & 0.99 & 0.08 & 1.93 & 0.09 & 1.96 \\
\hline 30 & -0.53 & Argillaceous limestone & 0.99 & 0.05 & 1.91 & 0.12 & 1.92 \\
\hline 30 & -0.45 & Argillaceous limestone & 0.95 & 0.05 & 1.91 & 0.01 & 2.01 \\
\hline 30 & -0.33 & Argillaceous limestone & 0.88 & 0.05 & 1.80 & 0.01 & 2.05 \\
\hline 29 & -0.31 & Argillaceous limestone & 0.80 & 0.05 & 1.54 & 0.02 & 1.93 \\
\hline 29 & -0.23 & Argillaceous limestone & 0.82 & 0.06 & 1.59 & 0.05 & 1.95 \\
\hline 29 & -0.20 & Argillaceous limestone & 0.88 & 0.05 & 1.75 & 0.02 & 1.99 \\
\hline 27 & -0.01 & Dolomitic limestone & 0.74 & 0.05 & 1.45 & 0.01 & 1.95 \\
\hline 26 & 0.12 & Argillaceous limestone & 0.84 & 0.05 & 1.63 & 0.00 & 1.95 \\
\hline $24 \mathrm{e}$ & 0.18 & Micritic limestone & 0.30 & 0.05 & 0.56 & 0.03 & 1.89 \\
\hline $24 \mathrm{e}$ & 0.24 & Micritic limestone & 0.57 & 0.02 & 1.09 & 0.08 & 1.98 \\
\hline
\end{tabular}




\begin{tabular}{llllllll}
$24 \mathrm{e}$ & 0.27 & Micritic limestone & 0.32 & 0.05 & 0.58 & 0.08 & 1.83 \\
$24 \mathrm{e}$ & 0.33 & Micritic limestone & 0.24 & 0.05 & 0.44 & 0.09 & 1.86 \\
$24 \mathrm{e}$ & 0.38 & Micritic limestone & 0.22 & 0.05 & 0.45 & 0.09 & 2.02 \\
$24 \mathrm{~d}$ & 0.41 & Micritic limestone & 0.36 & 0.05 & 0.67 & 0.10 & 1.89 \\
$24 \mathrm{~d}$ & 0.47 & Micritic limestone & 0.32 & 0.07 & 0.61 & 0.07 & 1.93 \\
$24 \mathrm{~d}$ & 0.56 & Micritic limestone & 0.27 & 0.07 & 0.50 & 0.07 & 1.81 \\
$24 \mathrm{~d}$ & 0.59 & Micritic limestone & 0.48 & 0.06 & 0.99 & 0.07 & 2.08 \\
$24 \mathrm{c}$ & 0.67 & Micritic limestone & 0.37 & 0.05 & 0.70 & 0.02 & 1.86 \\
$24 \mathrm{c}$ & 0.73 & Micritic limestone & 0.45 & 0.05 & 0.89 & 0.05 & 1.97 \\
$24 \mathrm{c}$ & 0.82 & Micritic limestone & 0.23 & 0.05 & 0.47 & 0.03 & 2.04 \\
$24 \mathrm{~b}$ & 0.85 & Micritic limestone & 0.74 & 0.05 & 1.50 & 0.02 & 2.03 \\
23 & 0.99 & Micritic limestone & 0.90 & 0.05 & 1.75 & 0.02 & 1.95 \\
23 & 1.22 & Micritic limestone & 0.93 & 0.07 & 1.81 & 0.09 & 1.96 \\
23 & 1.41 & Micritic limestone & 0.85 & 0.10 & 1.67 & 0.02 & 1.96 \\
23 & 1.42 & Micritic limestone & 0.94 & 0.05 & 1.87 & 0.04 & 1.99 \\
23 & 1.63 & Micritic limestone & 0.84 & 0.05 & 1.62 & 0.09 & 1.93 \\
23 & 1.73 & Micritic limestone & 0.86 & 0.05 & 1.72 & 0.05 & 2.00 \\
22 & 2.03 & Micritic limestone & 0.86 & 0.05 & 1.75 & 0.09 & 2.03 \\
22 & 2.48 & Micritic limestone & 0.77 & 0.05 & 1.51 & 0.04 & 1.95 \\
22 & 2.69 & Micritic limestone & 0.74 & 0.05 & 1.44 & 0.04 & 1.95 \\
22 & 3.64 & Micritic limestone & 0.73 & 0.05 & 1.39 & 0.05 & 1.91 \\
21 & 4.78 & Micritic limestone & 0.87 & 0.05 & 1.68 & 0.05 & 1.93 \\
19 & 6.32 & Micritic limestone & 0.83 & 0.05 & 1.66 & 0.07 & 2.01 \\
19 & 7.51 & Micritic limestone & 0.91 & 0.05 & 1.83 & 0.02 & 2.01 \\
\hline
\end{tabular}


Table DR3. Zn isotopic compositions of international standards analyzed during the course of this study.

\begin{tabular}{|c|c|c|c|c|c|c|c|}
\hline Name & Type & Session & $\begin{array}{c}\delta^{66} \mathrm{Zn} \\
(\%)\end{array}$ & $2 \mathrm{SD}$ & $\begin{array}{c}\delta^{68} \mathrm{Zn} \\
(\%)\end{array}$ & $2 \mathrm{SD}$ & Reference \\
\hline \multirow[t]{3}{*}{ BHVO-2 } & Basalt & 1 & 0.32 & 0.04 & 0.65 & 0.08 & This study \\
\hline & & & 0.33 & 0.04 & 0.65 & 0.06 & Chen et al. (2013) \\
\hline & & & 0.27 & 0.06 & - & - & Sossi et al. (2015) \\
\hline \multirow[t]{7}{*}{ BIR-1a } & Basalt & 1 & 0.25 & 0.03 & 0.49 & 0.05 & This study \\
\hline & & 2 & 0.29 & 0.05 & 0.56 & 0.02 & This study \\
\hline & & 3 & 0.29 & 0.05 & 0.54 & 0.07 & This study \\
\hline & & 4 & 0.28 & 0.02 & 0.53 & 0.02 & This study \\
\hline & & Average & 0.28 & 0.04 & 0.53 & 0.05 & This study \\
\hline & & & 0.31 & 0.04 & 0.61 & 0.06 & Chen et al. (2013) \\
\hline & & & 0.26 & 0.09 & 0.54 & 0.3 & Herzog et al. (2009) \\
\hline
\end{tabular}

$2 \mathrm{SD}=2$ times the standard deviation of the population of $\mathrm{n}$ repeat measurements of a sample solution. N: The times of repeat measurements of the same purification solution by MC-ICP-MS. 
Table DR4. Zn and Sr concentrations of the carbonate fraction of samples from the Meishan section, South China.

\begin{tabular}{|c|c|c|c|c|c|}
\hline $\begin{array}{l}\text { Depth to PTB } \\
\text { (m) }\end{array}$ & $\mathrm{Zn}(\mu \mathrm{g} / \mathrm{g})$ & $\operatorname{Sr}(\mu \mathrm{g} / \mathrm{g})$ & $\begin{array}{l}\text { Depth to } \\
\text { PTB (m) }\end{array}$ & $\mathrm{Zn}(\mu \mathrm{g} / \mathrm{g})$ & $\operatorname{Sr}(\mu \mathrm{g} / \mathrm{g})$ \\
\hline-12.03 & 17.5 & 317.3 & -0.45 & 19.3 & 181.4 \\
\hline-9.78 & 49.7 & 196.4 & -0.33 & 18.9 & 250.7 \\
\hline-8.29 & 46.0 & 171.1 & -0.23 & 13.5 & 311.8 \\
\hline-7.13 & 41.2 & 164.8 & -0.20 & 8.7 & 277.6 \\
\hline-5.63 & 37.3 & 252.2 & -0.01 & 6.6 & 272.0 \\
\hline-4.79 & 27.6 & 184.5 & 0.12 & 17.3 & 254.1 \\
\hline-4.63 & 35.7 & 193.2 & 0.18 & 50.2 & 274.0 \\
\hline-4.45 & 28.6 & 80.6 & 0.24 & 30.1 & 272.4 \\
\hline-4.14 & 39.1 & 229.2 & 0.27 & 49.3 & 293.2 \\
\hline-3.82 & 37.7 & 192.3 & 0.33 & 26.8 & 269.2 \\
\hline-3.66 & 36.1 & 177.2 & 0.38 & 106.5 & 309.2 \\
\hline-3.47 & 35.2 & 179.1 & 0.47 & 117.1 & 291.0 \\
\hline-3.24 & 32.1 & 142.8 & 0.59 & 17.8 & 141.7 \\
\hline-2.90 & 32.3 & 145.7 & 0.73 & 5.0 & 226.2 \\
\hline-2.71 & 22.6 & 214.9 & 0.93 & 0.2 & 243.3 \\
\hline-2.45 & 30.5 & 213.1 & 1.22 & 0.3 & 301.7 \\
\hline-2.07 & 28.0 & 118.1 & 1.33 & 0.3 & 317.3 \\
\hline-1.96 & 28.6 & 145.1 & 1.63 & 7.8 & 266.7 \\
\hline-1.77 & 27.8 & 206.5 & 1.73 & 0.3 & 367.1 \\
\hline-1.64 & 32.4 & 189.0 & 2.03 & 1.1 & 358.6 \\
\hline-1.52 & 28.0 & 171.3 & 2.48 & 0.1 & 294.9 \\
\hline-1.25 & 20.3 & 220.3 & 2.69 & 3.1 & 110.0 \\
\hline-1.10 & 33.5 & 236.6 & 3.64 & 0.2 & 364.3 \\
\hline-0.99 & 25.5 & 267.6 & 4.78 & 0.1 & 307.9 \\
\hline-0.89 & 35.8 & 231.4 & 6.32 & 1.8 & 263.7 \\
\hline-0.53 & 17.5 & 189.5 & 7.51 & 0.9 & 176.1 \\
\hline
\end{tabular}


Table DR5. Major elements (in wt.\%) of bulk sediments from the Meishan section.

\begin{tabular}{|c|c|c|c|c|c|c|c|c|c|c|c|}
\hline $\begin{array}{l}\text { Depth-to } \\
\text { PTB (m) }\end{array}$ & $\mathrm{SiO}_{2}$ & $\mathrm{TiO}_{2}$ & $\mathrm{Al}_{2} \mathrm{O}_{3}$ & $\mathrm{TFe}_{2} \mathrm{O}_{3}$ & $\mathrm{MnO}$ & $\mathrm{MgO}$ & $\mathrm{CaO}$ & $\mathrm{Na}_{2} \mathrm{O}$ & $\mathrm{K}_{2} \mathrm{O}$ & $\mathrm{P}_{2} \mathrm{O}_{5}$ & LOI \\
\hline \multicolumn{12}{|l|}{ Meishan } \\
\hline-12.031 & 25.01 & 0.34 & 6.69 & 2.83 & 0.13 & 3.75 & 29.67 & 0.07 & 1.82 & 0.06 & 29.04 \\
\hline-9.784 & 51.56 & 0.68 & 14.79 & 5.19 & 0.08 & 4.30 & 5.97 & 0.49 & 4.14 & 0.12 & 11.73 \\
\hline-8.287 & 53.96 & 0.74 & 14.79 & 4.56 & 0.06 & 3.98 & 5.12 & 0.42 & 4.12 & 0.10 & 11.21 \\
\hline-5.628 & 51.53 & 0.67 & 14.33 & 4.72 & 0.08 & 3.50 & 7.66 & 0.39 & 4.01 & 0.11 & 12.68 \\
\hline-4.634 & 53.93 & 0.69 & 14.58 & 5.66 & 0.05 & 3.48 & 5.10 & 0.41 & 4.07 & 0.08 & 10.99 \\
\hline-4.144 & 55.29 & 0.71 & 14.77 & 6.07 & 0.05 & 2.99 & 5.00 & 0.49 & 3.95 & 0.16 & 10.47 \\
\hline-3.655 & 52.82 & 0.71 & 14.27 & 5.61 & 0.07 & 4.23 & 6.69 & 0.52 & 3.52 & 0.08 & 10.5 \\
\hline-3.466 & 52.94 & 0.71 & 13.92 & 5.31 & 0.09 & 4.39 & 6.95 & 0.48 & 3.44 & 0.09 & 10.86 \\
\hline-2.713 & 37.28 & 0.42 & 9.05 & 4.42 & & 7.74 & 15.16 & & 2.39 & 0.08 & 22.46 \\
\hline-2.450 & 53.28 & 0.68 & 13.50 & 5.88 & 0.07 & 4.10 & 6.58 & 0.50 & 3.29 & 0.13 & 11.01 \\
\hline-2.073 & 52.26 & 0.65 & 13.71 & 5.76 & 0.07 & 4.05 & 6.12 & 0.44 & 3.70 & 0.11 & 12.24 \\
\hline-1.960 & 58.47 & 0.69 & 15.44 & 4.28 & 0.04 & 3.17 & 3.57 & 0.26 & 4.14 & 0.13 & 9.66 \\
\hline-1.772 & 58.11 & 0.70 & 14.74 & 5.25 & 0.04 & 3.00 & 3.81 & 0.23 & 3.94 & 0.11 & 9.67 \\
\hline-1.516 & 50.33 & 0.63 & 12.43 & 4.85 & 0.09 & 5.07 & 7.66 & 0.19 & 3.35 & 0.12 & 14.33 \\
\hline-1.095 & 54.31 & 0.72 & 13.58 & 4.61 & 0.08 & 4.65 & 6.33 & 0.10 & 3.80 & 0.09 & 10.82 \\
\hline Repeat & 53.63 & 0.69 & 13.71 & 4.61 & 0.07 & 4.55 & 6.04 & 0.15 & 3.71 & 0.09 & 12.93 \\
\hline-0.885 & 58.43 & 0.74 & 15.58 & 5.54 & 0.03 & 3.00 & 2.97 & 0.19 & 4.11 & 0.08 & 9.23 \\
\hline-0.531 & 44.06 & 0.50 & 10.23 & 3.90 & 0.13 & 6.84 & 12.02 & 0.14 & 2.72 & 0.08 & 19.28 \\
\hline-0.333 & 44.42 & 0.57 & 10.61 & 5.22 & 0.08 & 6.80 & 10.93 & 0.13 & 2.72 & 0.09 & 18.22 \\
\hline-0.226 & 38.25 & 0.52 & 9.09 & 4.32 & 0.08 & 6.76 & 15.89 & 0.22 & 2.13 & 0.11 & 21.72 \\
\hline-0.199 & 33.47 & 0.29 & 6.13 & 2.33 & 0.07 & 7.97 & 20.99 & 0.25 & 1.66 & 0.06 & 26.43 \\
\hline-0.019 & 27.48 & 0.20 & 4.06 & 2.10 & 0.09 & 5.01 & 30.58 & 0.07 & 0.98 & 0.01 & 28.80 \\
\hline 0.116 & 45.51 & 0.52 & 10.12 & 3.26 & 0.06 & 4.77 & 13.83 & 0.13 & 2.48 & 0.07 & 18.27 \\
\hline 0.239 & 14.22 & 0.05 & 1.45 & 0.94 & 0.10 & 0.78 & 45.33 & 0.07 & 0.47 & 0.18 & 35.83 \\
\hline 0.384 & 12.88 & 0.03 & 0.88 & 0.39 & 0.05 & 0.72 & 47.04 & 0.07 & 0.22 & 0.89 & 36.05 \\
\hline 0.586 & 35.62 & 0.10 & 3.01 & 1.20 & 0.04 & 0.91 & 30.02 & 0.12 & 0.64 & 0.05 & 27.51 \\
\hline 0.730 & 7.98 & 0.03 & 0.90 & 0.45 & 0.02 & 0.99 & 48.95 & 0.10 & 0.19 & 0.07 & 39.22 \\
\hline 1.217 & 7.08 & 0.03 & 0.97 & 0.37 & 0.01 & 0.75 & 49.68 & 0.03 & 0.25 & 0.10 & 39.92 \\
\hline 1.633 & 15.71 & 0.10 & 2.74 & 1.03 & 0.01 & 0.75 & 43.20 & 0.09 & 0.79 & 1.51 & 33.48 \\
\hline 2.482 & 31.89 & 0.09 & 2.85 & 0.78 & 0.01 & 1.55 & 32.89 & 0.04 & 0.61 & 0.47 & 27.77 \\
\hline 3.641 & 1.19 & 0.00 & 0.20 & 0.04 & 0.01 & 0.66 & 54.31 & 0.02 & 0.05 & 0.16 & 42.71 \\
\hline 4.782 & 5.44 & 0.00 & 0.19 & 0.15 & 0.01 & 0.66 & 51.23 & $<0.03$ & $<0.03$ & 0.18 & 41.6 \\
\hline
\end{tabular}




\section{References}

Burgess, S.D., Bowring, S., and Shen, S.Z., 2014, High-precision timeline for Earth's most severe extinction: Proceedings of the National Academy of Sciences of the United States of America, v. 111, P. 3316-3321, doi: 0.1073/pnas.1403228111.

Cao, C.Q., Love, G.D., Hays, L.E., Wang, W., Shen, S.Z., and Summons, R.E., 2009, Biogeochemical evidence for euxinic oceans and ecological disturbance presaging the end-Permian mass extinction event: Earth and Planetary Science Letters, v. 281, p. 188-201, doi:10.1016/j.epsl.2009.02.012.

Chen, H., Savage, P.S., Teng, F.-Z., Helz, R.T., and Moynier, F., 2013, Zinc isotope fractionation during magmatic differentiation and the isotopic composition of the bulk Earth: Earth and Planetary Science Letters, v. 369-370, p. 34-42, doi: 10.1016/j.eps1.2013.02.037.

Grice, K., Cao, C.Q., Love, G.D., Bottcher, M.E., Twitchett, R.J., Grosjean, E., Summons, R.E., Turgeon, S.C., Dunning, W., and Jin, Y.G., 2005, Photic Zone Euxinia During the Permian-Triassic Superanoxic Event: Science, v. 307, p. 706-709, doi:10.1126/science.1104323.

Herzog, G.F., Moynier, F., Albarede, F., and Berezhnoy, A.A., 2009, Isotopic and elemental abundances of copper and zinc in lunar samples, Zagami, Pele's hairs, and a terrestrialbasalt: Geochimica et CosmochimicaActa, v. 73, p. 5884-5904, doi: 10.1016/j.gca.2009.05.067.

Jiang, H.S., Lai, X., Luo, G., Aldridge, R., Zhang, K., and Wignall, P.B., 2007, Restudy of conodont zonation and evolution across the P/T boundary at Meishan section, Changxing, Zhejiang, China: Global Planetary Chang, v. 55, p. 39-55, doi:10.1016/j.gloplacha.2006.06.007.

Jin, Y.G., Shen, S.Z., Henderson, C.M., Wang, X.D., Wang, W., Wang, Y., Cao, C.Q., and Shang, Q.H., 2006, The Global Stratotype Section and Point (GSSP) for the 
boundary between the Capitanian and Wuchiapingian stage (Permian): Episodes, V. 29 , no. 4 , p. 253-262.

Kunzmann, M., Halverson, G.P., Sossi, P.A., Raub, T.D., Payne J.L., and Kirby, J., 2013, $\mathrm{Zn}$ isotope evidence for immediate resumption of primary productivity after snowball Earth: Geology, v. 41, p. 27-30, doi:10.1130/G33422.1.

Liu, S.-A., Li, S.G., He, Y.S., and Huang, F., 2010, Geochemical contrasts between early Cretaceous ore-bearing and ore-barren high-Mg adakites in central-eastern China: implications for petrogenesis and $\mathrm{Cu}-\mathrm{Au}$ mineralization: Geochimica et CosmochimicaActa, v. 74, p. 7160-7178, doi:10.1016/j.gca.2010.09.003.

Liu, S.-A., Li, D., Li, S., Teng, F.-Z., Ke, S., He, Y., and Lu, Y., 2014, High-precision copper and iron isotope analysis of igneous rock standards by MC-ICP-MS. Journal Analytical Atomic Spectrometry, v. 29, p. 122-133, doi:10.1039/c3ja50232e.

Little, S.H., Vance, D., Walker-Brown, C., and Landing, W.M., 2014, The oceanic mass balance of copper and zinc isotopes, investigated by analysis of their inputs, and outputs to ferromanganese oxide sediments: Geochimica et CosmochimicaActa, v. 125, p. 673-693, doi: 10.1016/j.gca.2013.07.046.

Lv, Y.W., Liu, S.A., Zhu, J.M., and Li, S.G., 2016, Copper and zinc isotope fractionation during deposition and weathering of highly metalliferous black shales in central China: Chemical Geology, v. 422, p. 82-93, doi:10.1016/j.chemgeo.2015. 12.017.

Maréchal, C.N., Telouk, P., and Albarède, F., 1999, Precise analysis of copper and zinc isotopic compositions by plasma-source mass spectrometry: Chemical Geology, v. 156, p. 251-273, doi:10.1016/S0009-2541(98)00191-0.

Moeller, K., Schoenberg, R., Pedersen, R.-B., Weiss, D., and Dong, S., 2012, Calibration of the new certified reference materials ERM-AE633 and ERM-AE647 for copper and IRMM-3702 for zinc isotope amount ratio determinations: GeostandardsGeoanalytical Research, v. 36, p. 177-199, 
doi:10.1111/j.1751-908X.2011.00153.x.

Nabbefeld, B., Grice, K.,Schimmelmann, A., Sauer, P.E.,Böttcher, M.E., and Twitchett, R., 2010, Significance of $\delta D_{\text {kerogen, }}, \delta^{13} C_{\text {kerogen }}$ and $\delta^{34} S_{\text {pyrite }}$ from several Permian/Triassic (P/Tr) sections: Earth and Planetary Science Letters, v. 295, p. 21-29, doi:10.1016/j.eps1.2010.03.015.

Pichat, S., Douchet, C., and Albarède, F., 2003, Zinc isotope variations in deep-sea carbonates from the eastern equatorial Pacific over the last 175 ka: Earth and Planetary Science Letters, v. 210, p. 167-178, doi:10.1016/S0012-821X (03)00106-7.

Shen, S.Z., and 21 others, 2011, Calibrating the End-Permian Mass Extinction: Science, v. 334, p. 1367-1372, doi:10.1126/science.1213454.

Sossi, P.A., Halverson, G.P., Nebel, O., and Eggins, S.M., 2015, Combined separation of $\mathrm{Cu}, \mathrm{Fe}$ and $\mathrm{Zn}$ from rock matrices and improved analytical protocols for stable isotope determination: GeostandardsGeoanalytical Research, v. 39, p. 129-149, doi:10.1111/j.1751-908X.2014.00298.x.

Vance, D., Little, S.H, Archer, C., Cameron, V., Andersen, M.B., Rijkenberg, M.J.A., and Lyons, T.W., 2016, The oceanic budgets of nickel and zinc isotopes: the importance of sulfidic environments as illustrated by the Black Sea. Phil. Trans. R. Soc. A 374: 20150294, doi: org/10.1098/rsta.2015.0294.

Wu, H.C., Zhang, S.H., Hinnov, L.A., Jiang, G.Q., Feng, Q.L., Li, H.Y., and Yang, T.S., 2013, Time-calibrated Milankovitch cycles for the late Permian: Nature Communications, v. 4, no. 2452, doi: 10.1038/ncomms3452.

Yin, H., Zhang, K., Tong, J., Yang, Z., and Wu, S., 2001, The global Stratotype section and point (GSSP) of the Permian-Triassic boundary: Episodes, v. 24, no. 2, p. 102-114. 
Yuan, D.X., Shen, S. Z., Henderson, C.M., Chen, J., Zhang, H., and Feng, H. Z., 2014, Revised conodont-based integrated high-resolution timescale for the Changhsingian Stage and end-Permian extinction interval at the Meishan sections, South China: Lithos, v. 204, p. 220-245, doi:10.1016/j.lithos.2014.03.026.

Zhang, K.X., Lai, X.L., Tong, J. N., and Jiang, H. S., 2009, Progresses on study of conodont sequence for the GSSP section at Meishan, Changxing, Zhejiang province, South China: ActaPalaeontol. Sin, v. 48, p. 474-486. In Chinese with English abstract.

Ziegler, A.M., Hulver, M.L., and Rowley, D.B., 1997, Permian world topography and climate, in Martini, I.P., ed., Late glacial and postglacial environmental changes--Quaternary, Carboniferous-Permian and Proterozoic:New York, OxfordUniversity Press, p. 111-146. 